\title{
MEASUREMENT CORRECTION OF TWO-DIMENSIONAL WIND TUNNEL WITH POROUS WALLS USING SINGULARITY METHOD
}

\section{TAHA AHMED ABDULLAH \& MOHAMMED SALEH MOHAMMED}

Mechanical Department, Engineering College, Mosul University, Mosul, Iraq

\begin{abstract}
The correction method is developed to correct the distribution of pressure coefficient around an airfoil for the aim of the post processing of measurement data from wind tunnel. The measured pressure coefficient is numerically corrected to compensate walls interference effects for wind tunnel test section. The airfoil NACA0012, in this paper, is simulated by segments with linear vortex, which are then mirrored due to the floor and ceiling of the wind tunnel test section using sufficient number of images, to simulate the flow around an airfoil in the test section with walls. The perforated wall is approximated by constant source segments. Flow calculations, both in the free stream and with the presence of tunnel walls, are then performed. The numerically pressure coefficient difference obtained between these two cases should be superimposed to the measured pressure coefficient distribution in the wind tunnel for the same airflow conditions and corresponding points, resulting in the experimental pressure coefficient distribution corrected. With the relative height reduction of the wind tunnel test section, the correction values increase. The verification of lift coefficient corrections in this paper is concentrated, where very good agreements have been obtained comparison with several well-known analytical methods.

KEYWORDS: Coefficient of Pressure; Method of Singularity; Vortex Segment; Interference Wall; Corrections of Wind Tunnel; Porous Walls
\end{abstract}

Received: Mar 03, 2020; Accepted: Mar 23, 2020; Published: Jun 30, 2020; Paper Id.: IJMPERDJUN2020256

\section{INTRODUCTION}

In wing forming and design an airfoils cross-sectional shapes are applied; whose aerodynamic characteristics effect the characteristics of flying aircraft. Aerodynamic parameters of a wing of an infinite span are defined as airfoil characteristics, where is the two-dimensional (2D) flow, since no influence forthe secondary wing tip flow, due to which the real - finite span (i.e. finite aspect ratio) wings are exposed to 3D flow patterns. Namely, due to the difference ofpressure between lower and upper the surface of wing, whichresults lift force, on tips the flow "leaks" to upper side from lower around them, pushing the flow towards on upper wing surface, and on lower side away from the wing root. Once the airfoils aerodynamic characteristics that will be used in wing design will be known, actual finite aspect ratio wing characteristic can nowadays be calculated by different available methods.

In wind tunnels characteristics of airfoil are usually determined, or at least by wind tunnels confirmed, if the airfoil analysis and design had been determined by computational methods. In wind tunnels the 2D flow is represented by putting a rectangular wing with constant airfoil model between the side walls oftest section, or between boundary plates, which physically prevent secondary flow around the model's tips. The results obtained in wind tunnels considering 2D airfoil. The subject of this paper is the corrections of results which experimentally obtained due to finite test section size. With wind tunnel walls all the flow field about the airfoil affect, considering 
the flow curvature in front and behind the airfoil, pressure distributions and speed, etc. In this paper, two relative height for two different wind tunnels are considered i.e. two kinds of test sections, so that the required corrections can be directly subtracted from or added to the measured flow quantities. The influence in 2D analyses, of side walls can be neglected because the most perturbances generated in normal and longitudinal directions both by the airfoil and walls on the flow characteristics.

For computational analyses, the difference betweendetermined values for an airfoil of flow parameters first with, and withouteffect of wind tunnel walls, is combined with coefficient of pressure distributions measured in wind tunnel, to obtain experimental datawith correction, which to free stream conditionscorrespond.

\subsection{Correction Approaches:}

A simulation of airfoil by singularity method is used to determine the wind tunnel wall interference [1].Correction method is represented to correct test model deformation effects due to aerodynamic loads in transonic wind tunnel [2]. In reference [3], the flow around airfoil computationally solved and the solution is used to calculate the curvature of the adaptive wall which will in the same pressure distribution result over the wall as the distribution from calculationsobtained. In adaptive slots the same principal idea using is described in [4]. Sawada in [5] interference effects calculate of wind tunnel withventilated walls, horseshoe vortex distribution used over a wing. For boundary condition, he used measured pressure distribution over walls for the solution of potential flow within test section. Mokry [6] solved Laplace equation byusing fast Fourier transformation as boundary conditions in two-dimensional wind tunnel test section, to solve Laplace's equation using a doublet-vortex approximation of the airfoil shape combined withmeasured wind tunnel wall distributions ofpressure. The method of wall correction of Kuppers [7] is based on pressure distribution measured on the walls oftunnel to solve Laplace's equation. The experimental data in this method combines with theoretical calculated boundary conditions. The wind tunnel wall interference calculation is based on the Greens integral solution. Mokry [8] used the method of first order doublet-panel to correct angle of attack and Mach number obtained by measurements in the wind tunnel with perforated wallstest section. The measured forces on the model and measured static pressure over walls are applied as boundary conditions. The procedure by Thomas and Zeki [9] uses pressure distribution measurements on the wall to develop a flow field solution based on the singularitymethod. As a pressure boundary condition this flow field solution is then imposed in a CFD simulation of the internal flow field. For two and three-dimensional wind tunnels with porous walls the singularity method is applied. Ashil and Week [10] the Greens theorem used to solve Laplace's equation to simulate a potential flow field. For two- and three-dimensional model the wall interferences are calculated by static pressures measuredas boundary conditions on the walls. Holt and Hant [11] calculated theeffects of subsonic wall interference in both twoand three-dimensional model by dividing the ceiling and floor with linear distribution of vorticity panels. Horsten and Veldhuis [12], present a method based on uncorrected wind tunnel measurements and fast calculation techniques.

In this paper, the airfoil in the test section of a wind tunnelis simulated with large number of panels each panel is represented with linear vortex strength, and the source panels with constant strength are used to approximate the perforated wind tunnel walls. To simulate the presence of wind tunnel walls, the panels are repeatedly mirrored due to the floor and ceiling of the wind tunnel test section, thus approximating real flow conditions in test section as a result of linear vorticity strength variation and constant sources panel effect. over the airfoil, thePressure distribution is calculated both, with the wall presence (simulated by images), and without images (free stream). Thepressure coefficients difference between numerically calculated for free-stream solution and numerical solution with walls presence iscalculated, and superimposed to measured pressure distributions, in an actual wind tunnel. Correction pressure distribution approach corrects the 
coefficient oflift, as well as the coefficient ofmoment. In pressure coefficient correction,buoyancy effect is contained, so measured speed andangle of attack is true speed and angle of attack. In this paper, the corrections are concentrated on the coefficient of lift evaluations. The evaluations have been carried out for symmetrical airfoil NACA 0012, used as standard "test" airfoil for many years for the investigation of both the experimental facilities and computational models.

Also, presented method, based on the mirroring of image below and above the wind tunnel airfoil, confines the corrections to the perforated walls condition. The difference between her applied model and most of the previously developed methods is that it applies corrections to the coefficient of pressure, while keeping the same Mach number and angle of attack.

\section{Essential Assumptions}

The Following Assumptions are adopted in This Paper:

- The flow is irrotational and inviscid around a 2D airfoil.

- The airfoil is approximated by a large number of panel's withlinear vortex.

- Subsonic air flow.

- Corrections are small, and can be linearly applied.

\subsection{Governing Equations}

The equation ofpotential flow replaces Navier-Stokes equations as mentioned in thefirst assumption:

$$
\beta^{2} \frac{\partial^{2} \widehat{\emptyset}}{\partial \hat{x}^{2}}+\frac{\partial^{2} \widehat{\emptyset}}{\partial \hat{y}^{2}}
$$

The correction parameter of compressibility is defined as:

$$
\beta=\sqrt{1-M^{2}}
$$

where $M$ is the Mach number offree- stream, and the velocity potential of small disturbance perturbation $\widehat{\emptyset}$ has been defined as follows:

$$
\widehat{\emptyset}^{-}=U_{\infty} x+U_{\infty} y+\emptyset
$$

Transforming $\widehat{\varnothing}$ and $(\hat{x}, \hat{y})$ by equation:

$$
(x, y)=(\hat{x}, \beta \hat{y})
$$

we obtain, $\Delta \emptyset=0$ where Laplace operator is:

$$
\Delta=\frac{\partial^{2}}{\partial \hat{x}^{2}}+\frac{\partial^{2}}{\partial \hat{y}^{2}}
$$


For free-stream conditions, this equation is solved, and for airfoil in the test section of wind tunnel, using the principle of superposition of singular solutions, since the equation ofLaplace is linear. Any combination of singular solutions is also the solution of the Laplace's equation. Our task here is to select arbitrary constants for singularity solutions that, as well as satisfying the equation Laplace, also satisfying the boundary conditions.

\subsection{Boundary Conditions}

On both wind tunnel walls and airfoil, at any point of the solid surface the normal component of the velocity must be equal to zero. This requirement is achieved by:

1) An imaging system of the airfoil has been established, represented by panels with linear vortex, due to the ceiling and floor of the test section of wind tunnel. This imaging system encloses simulation of streamlines for real flow-field, which are parallel to the test section ceiling and floor.

2) Establishingthat, over the solid surface of the airfoil the normal component of the velocities (i.e. on the panels control points) the following condition has beensatisfied:

$$
V_{i} \cdot \vec{n}_{i}=0
$$

Subscript $i$ a control point indication whose coordinates are $x_{c i}, y_{c i}$

3) To enclosetrailing edge velocity is finite; at the trailing edge the Kutta condition must be satisfied.

\subsection{Numerical modeling for ventilated wall wind tunnel}

\subsubsection{Fundamental ideas of this paper for 2D wind tunnel wall corrections for ventilated wall test sections}

Again, correction procedure is applicable for both cases when distribution of pressure is measured around airfoil or when only aerodynamic coefficients $C_{L}$ and $C_{M}$ are measured. Airfoil is again represented by a system of straight elements, with linearly varying vortices strength. Nodal intensities of the vortices are determined by requiring that cross-flow through control point does not exists. Wind tunnel walls are ensured by multiple mirroring of complete airfoil resulting in exact solid wall boundary condition. Additionally, source/sink singularity panels are distributed along wind tunnel walls which simulate wall porosity. Intensity of the sources/sinks is proportional to the pressure difference between wind tunnel test section and plenum chamber. Coefficient of the proportionality is determined by comparison of pressure distributions obtained by numerical calculations and that obtained by measurement.

If only aerodynamic coefficients are measured, then comparison is done between measured lift coefficient and calculated lift coefficient.

Proportionality coefficient is determined properly if numerical calculation agrees well with measurement. Corrections to lift coefficient and to moment coefficient are determined the same as for the solid wall case. The correction procedure for ventilated 2D wind tunnel test section illustrates Figure 1. 


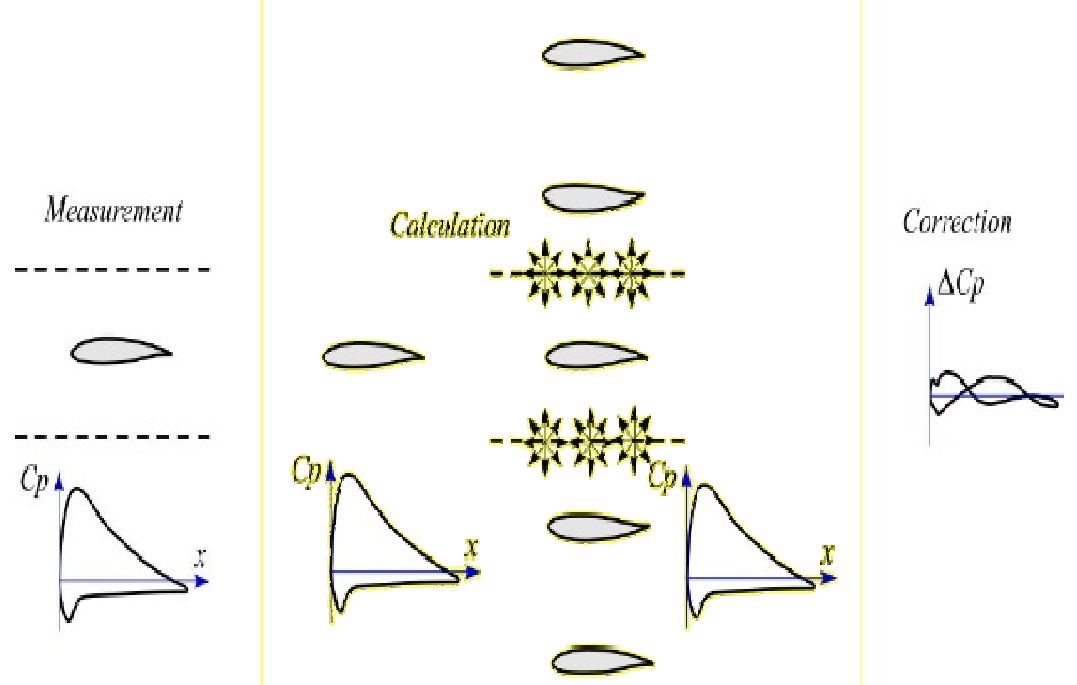

Figure 1: 2-D Wind Tunnel Correction Procedure For Ventilated Walls.

\subsubsection{Fundamental Assumptions}

The following assumptions are adopted:

- The flow is incompressible (compressibility is taken over the parameter $\beta=\sqrt{1-M^{2}}$

- The effects of porous walls can be superimposed the solution with the solid walls.

- The porosity of one wall does not affect the porosity of the second, in the calculation sense.

\subsubsection{Application of Bernoulli equation}

The plenum chamber around the model in wind tunnel is usually at the same pressure as the flow pressure in front of the model (far enough). If the pressure and velocity of flow far enough from the model front are denoted as $P_{\infty}, V_{\infty}$, then the Bernoulli equation will be;

$$
\begin{aligned}
\frac{1}{2} \rho V_{\infty}^{2}+p_{\infty}= & \frac{1}{2} \rho V^{2} \\
& +p
\end{aligned}
$$

And after rearranging

$$
p-p_{\infty}=\frac{1}{2} \rho V_{\infty}^{2}\left(1-\frac{V^{2}}{V_{\infty}^{2}}\right)
$$

If we assume that the velocity at the walls wind tunnel can be written as:

$$
V=V_{\infty}+u
$$

then substituting it in the previous equation we get: 


$$
p-p_{\infty}=\frac{1}{2} \rho V_{\infty}^{2}\left(1-\frac{V_{\infty}^{2}-2 V_{\infty} u+u^{2}}{V_{\infty}^{2}}\right)
$$

Also, after neglecting $u^{2}$ in comparison with other terms:

$$
p-p_{\infty}=\rho V_{\infty} u
$$

\subsubsection{Boundary Condition for a Ventilated Wall}

It is eligible to formulate the ventilated wall boundary condition in order to generalize the approach adopted in the experiments. It is alsoeligible to find a basis for comparison between interference due to an 'ideal' slotted-ventilated wall (i.e., with inviscid flow) and interference when viscous slot flow is important. The porosity of the boundaryin both cases influences the interference up-wash in the wind tunnel, but an ideal ventilated wall the porosity relates the pressure drop across the wall to mass flow through it in inviscid flow, whilefor slotted wall the effective porosity is significant only when viscous flow is predominant at the boundary, e.g., when the boundary-layer displacement thickness larger thanslot width. Due to viscous slot flow the porosity can be joined with truly porous wall and one might a similar porosity effect expect for a tunnel with ventilated wall if the size ofperforation is less than the displacement thickness ofboundary-layer [15]. In [17] the outflow to the pressure drop across the wall is related. The pressure drop across a porous wall is given by Darcy's law:

\section{$\Delta p \propto w$}

$$
\Delta p=\frac{\rho V_{\infty}}{P} w
$$

Where $\mathrm{P}$ is the porosity factor.

The boundary condition obtains by Goodman at the porous wall from Equations (9) and (10).

$$
w=P u=P\left(V-V_{\infty}\right)
$$

Where the $w$ and $u$ are the vertical and axial component of induced velocity, $V_{\infty}$ is the free velocity of flow far from the model.

In inviscid flow before determinationthe porosity parameter for the slotted-ventilated walls, first the boundary condition must beconsideredfor a ventilated wall. Reference [18] in incompressible flow shows that the relation between the velocity normal to the wall and the perturbation velocity in the stream direction is:

$$
w=P_{1} u
$$

where $P_{I}$ is a porosity constant for a wall configuration.

From Equation (13) we start to calculate the interference of wind tunnel with porosity walls. The axial velocity on the walls $u$ is calculated from the numerical solution of the effects of linear vortex panels and their images inside wind tunnel on the solid walls. This velocity is regarded as initial value to solve the equations of constant strength source which are taken in case of ventilated wind tunnel walls. 


\subsubsection{The Effect of Constant Strength Sources in the Wind Tunnel Walls Panels}

In the previous modeling [1] we considered the solid walls interference in the testing model inside test section of wind tunnel. In this modeling: the wind tunnel walls are divided with $n$ panels and each panel is approximated with constant strength source to model the porosity effects through the wind tunnel walls as shown in Figure 2.

For each panel the induced velocities are calculated in the control point for the same panel without contribution of the other panels of constant strength sources, because the panels in the same straight line cannot induce velocity in the point which the line of velocity normal to the same straight line. The effect of constant strength source panels on upper wall does not affect porosity lower wall panels, and vice versa.

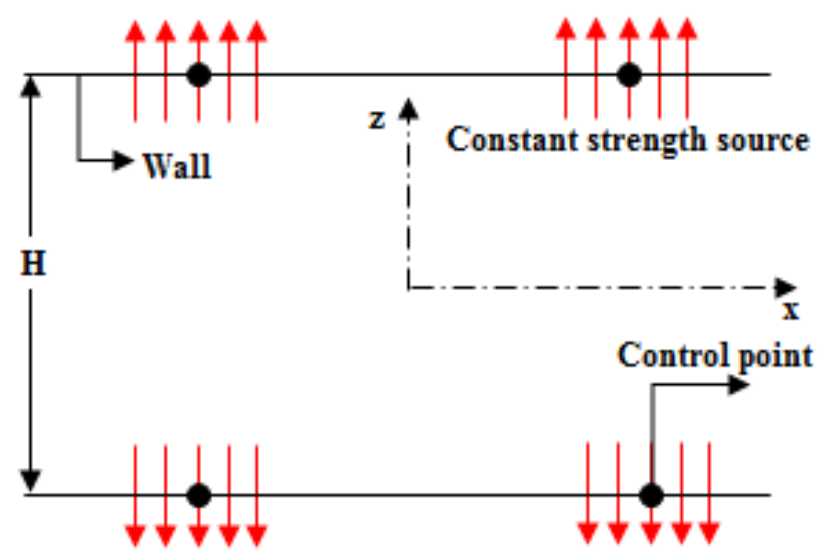

Figure 2: Constant Strength Source Panels on Wind Tunnel Walls.

The induced velocities for constant strength source can be calculated from the equations below:

$$
\begin{aligned}
& u=\frac{\sigma}{4 \pi} \ln \frac{\left(x-x_{1}\right)^{2}+z^{2}}{\left(x-x_{2}\right)^{2}+z^{2}} \\
& w=\frac{\sigma}{2 \pi}\left(\tan ^{-1} \frac{z}{x-x_{2}}-\tan ^{-1} \frac{z}{x-x_{1}}\right)
\end{aligned}
$$

Where $\sigma$ is the source strength. The control points of the constant strength source panels are defined by coordinates:

$$
x_{c i}=\frac{x_{2}-x_{1}}{2}+x_{1} z_{c i}=\frac{z_{2}-z_{1}}{2}+z_{1}
$$

If the induced velocities at control points are calculated from the constant strength sources, positioned on the same height, then the local vertical axis coordinate for all of them is $\mathrm{z}=0$, (see Fig.3). This means that the normal component of induced velocity at a panel control point Eq. (17) is affected only by its own panel, and takes the form:

$$
w(x)= \pm \frac{\sigma(x)}{2}
$$




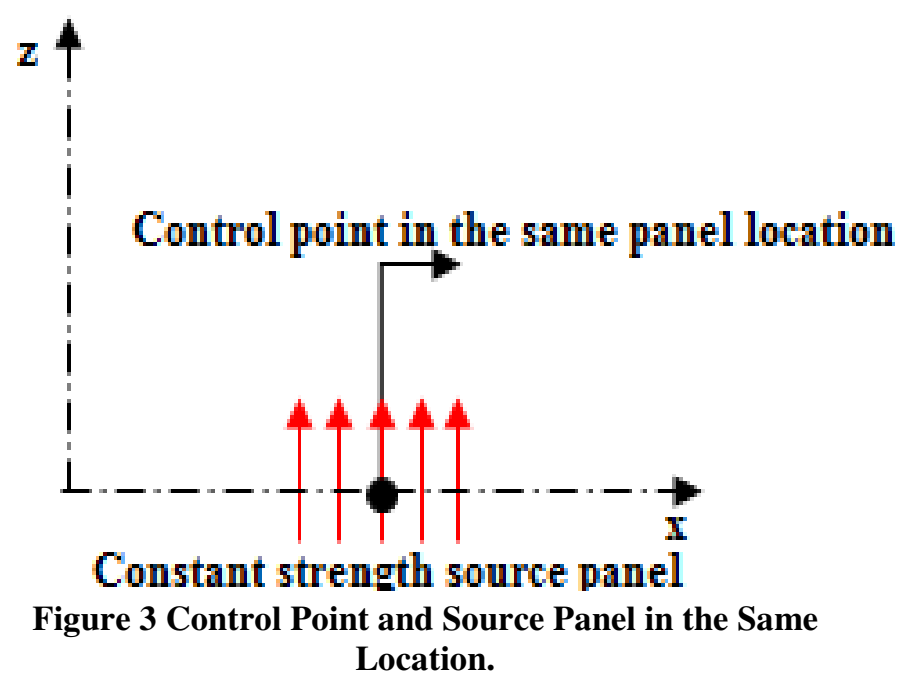

The sign plus or minus in the previous equation is important to distinguish between the conditions when the panel is approached from its upper or from its lower side.

The source strength can be calculated from the Equations (13) and (18) as:

$$
\sigma=2 P u=2 P\left(V-V_{\infty}\right)
$$

In the calculation of source strength (sigma), the value of the axial velocity on the wall $V$ is taken from the numerical calculation of the influence of the airfoil's linear vortex panels on the upper and lower solid wall control points of wind tunnel [1]. Each wall control point on the wind tunnel walls is affected by all airfoil's panels of linear strength vortices. The value of porosity parameter $P$ must be estimated depending on the procedure correction of the measured data in the wind tunnel i.e. iterate the value of porosity factor in the numerical calculation until the results be equivalent to the measured wind tunnel data.

After calculation the strength source panel, the induced velocity in the panels control points of the lower and upper walls can be calculated easily from the Eqs. (15) and (17).

\subsubsection{The Effect of Sources Panels on the Vortex Panel Control Points on the Airfoil}

As already mentioned, the induced velocity in control points of constant sources panels on the walls are calculated only as self-influence, without contribution of the other panels in the calculation. Also, the airfoil inside test section of the wind tunnel was approximated with $n$ panels of linear vortex strength segments and the numerical solution is carried out for free stream (without walls effect i.e. without image system calculation), and with wind tunnel walls effect (in this case with image system calculation) [1]. As a result, the pressure distributions and lift coefficients are obtained.

Now the effects between the linear strength vortices on the approximated airfoil and constant strength sources on the wind tunnel upper and lower walls must be considered.

All the source panels for the upper and lower walls induce velocity at each control point of the airfoil's vortex panel, therefor the velocity in the control point of the linear strength vortex panel is a summation of two velocities, induced from linear vortex panels and constant source panels see Figure .4. The velocity induced from vortex panel was numerically 
calculated and presented in [1]. Also, the detailed numerical simulation of the flow field around airfoil can be found in reference [1]. The additional velocity in the control point of vortex panel as a result of the constant source panel's effects was calculated from Eqs. (16) and (17).

Figure 4 shows The system of equations which were solved in case of solid walls to calculate the vortex strength parameter, now takes another form therefore it must be solved for the second time to calculate the induced velocities, i.e. the additional influence of source panels at test section walls. As a result, the pressure distribution and lift coefficients are obtained.

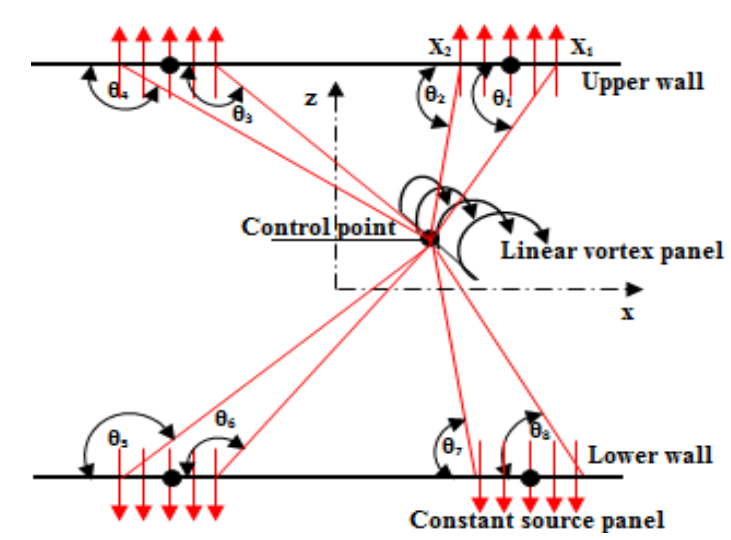

\section{Figure 4 Source Panels Induced Control Points}

In Linear Vortex Panels.

\subsection{Sources of Experimental Data for Calculations of Test Sections with Ventilated Walls}

Verifications of the calculation model for the case of ventilated walls will be verified referencing experimental data from two relevant experimental facilities, the T-38 Transonic wind tunnel VTI Žarkovo - Belgrade andTransonic cryogenic tunnel (0.3-m NASA Langley TCT).

\subsubsection{T-38 Wind Tunnels (VTI Žarkovo, Belgrade)}

Wind tunnel tests were performed in the test section $0.38 \times 1.5 \mathrm{~m}$ using the NACA 0012 calibration model in the subsonic and transonic Mach number range. Pressures about the airfoil were measured with two Scanivalves. Wing pressure holes 1 to 40 inclusive were connected to the Scanivalve 1, while ports 41 to 80 inclusive were connected to the Scanivalve 2. The two additional Scanivalves were used to measure pressures on upper and lower wind tunnel walls [19].

\subsubsection{Transonic Cryogenic Tunnel (0.3-m NASA Langley TCT)}

The test sections (various sizes of test sections can be used) are rectangular, have slotted bottom and top walls and solid sidewalls.In each of these walls two slots are located with a spacing of 4.0 in $(10.16 \mathrm{~cm})$. All modelsurface and tunnel ceiling, and floor pressures were measured using 48-port Scanivalves, to high precision variable capacitance type pressure transducersconnected. The test program considered in this paper was conducted in the 8 in by 24 in $(20.32 \mathrm{~cm}$ by 60.96 $\mathrm{cm}$ ) two dimensional test section of the Langley 0.3-meter transonic cryogenic tunnel to obtain the characteristics ofaerodynamic of a series of 2D airfoils (including NACA 0012), at transonic and subsonic speeds and flight-equivalent Reynolds numbers [16]. 


\section{RESULTS AND DISCUSSIONS}

\subsection{Wind Tunnel T-38}

The numerical calculations were carried out for the NACA0012 airfoil at $\alpha=2^{\circ}, 4^{\circ}, 6^{\circ}$ and $\mathrm{M}=0.3$, corresponding to one of the test cases in T-38 wind tunnel.

The pressure coefficient distributions measured on the NACA0012 airfoil in the T-38 test section and the numerically calculated values of pressure coefficient for same airfoil are shown in Figure 5.

The differences between measured and numerically calculated distributions of pressure coefficient are small, and they can be contributed partially to the neglected viscous effects within the calculation method, and partially to inevitable small measurement errors. Those results verify the introduced and applied method of ventilated walls modeling, and their influence on the calculated pressure distributions about the airfoil in the simulated test section.

The next step was numerical determination of required corrections, quantified as difference between pressure coefficient distributions between previously calculated values in ventilated test section, and the free stream distributions. Pressure coefficient distribution about the NACA0012 airfoil, approximated by linear vortices strength panels, is numerically calculated this time with number of mirrored images set to zero (walls excluded), and with zero strength of sources/sinks (ventilation effects excluded). The pressure coefficient distributions for these two cases are compared and shown in Figure 6.

The differences between the two pressure coefficient distributions at control points $i$, denoted as $\Delta C_{p i}$, represent the values of calculated corrections.

Finally, the measured pressure coefficient distributions in T-38 wind tunnel are corrected by superimposing $\Delta C_{p i}$ to them. For that purpose, it is necessary to interpolate the $\Delta C_{p i}$ values, calculated at panel control points, to positions which corresponds pressure measurement.

The corrections applied to T-38 measurements are shown in fig, while integrated values of corrections, as global parameters, are shown in Tab.1, and Figure 7.

Those results indicate that the influence of ventilated walls on the pressure coefficient distribution is relatively small, primarily because of the large relative height of test section $h=6$ applied in the T-38 tunnel, and thus also applied in the calculations. Table 1 shows that, with the increase of angle of attack, corrections have slight increasing tendency. Table 1 also shows good agreements of lift coefficients obtained in wind tunnel ( $C_{L}$ measured), and by numerical calculations (denoted as $C_{L}$ with wall effect), verifying the here applied method of ventilated walls numerical modeling.

Table 1 since lift coefficient corrections are obtained by subtracting calculated free stream values from values with wall effect; they are all of negative sign. Thus when subtracted from wind tunnel lift coefficients, they give corrected values which are larger than measured. 


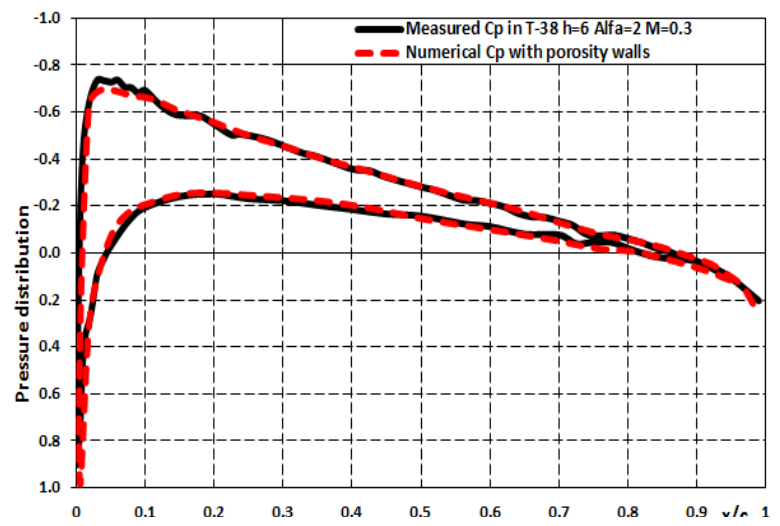

Figure 5: Measured and Calculation Pressure Distribution in T-38 Wind Tunnel.

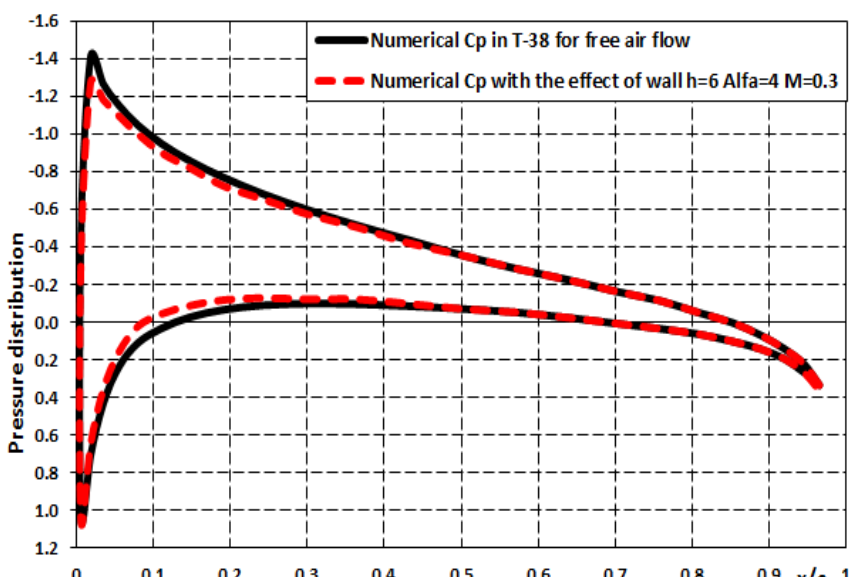

Figure 6: Numerical $C_{p}$ for Wind Tunnel Wall Effect and for Free Stream T-38.

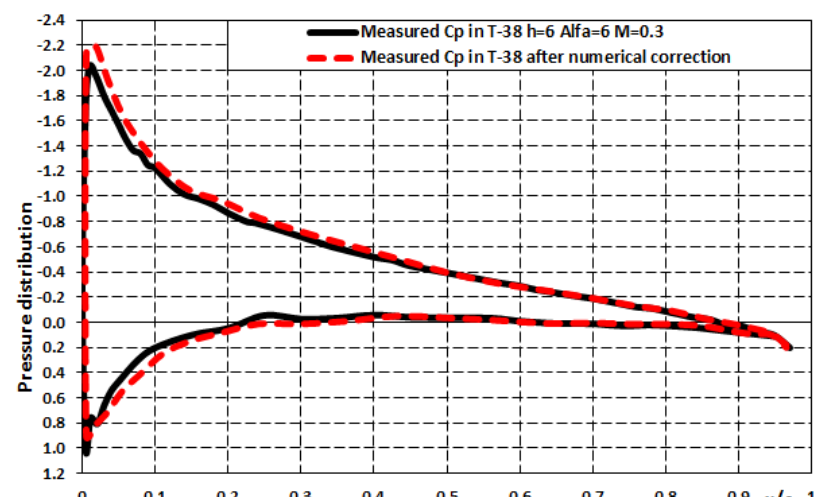

Figure 7: Measured Cp After Correction In T-38 Wind Tunnel.

Table 1: Lift Coefficient Correction for T-38 Wind Tunnel

\begin{tabular}{|c|c|c|c|c|c|}
\hline \multicolumn{5}{|c|}{ For T-38 Wind Tunnel With Height $\boldsymbol{H}=\mathbf{6}, \mathbf{M}=\mathbf{0 . 3}$} \\
\hline Alfa & $\mathbf{C}_{\mathbf{l}}$ with Wall Effect & $\mathbf{C}_{\mathbf{l}}$ for Free Stream & $\Delta \mathbf{c}_{\mathbf{l}}$ & $\mathbf{C}_{\mathbf{l}}$ Measured & C $_{\mathbf{l}}$ After Correction \\
\hline $2^{\mathrm{o}}$ & 0.1931 & 0.2475 & -0.0544 & 0.1900 & 0.2444 \\
\hline $4^{\mathrm{o}}$ & 0.3970 & 0.4944 & -0.0974 & 0.3860 & 0.4834 \\
\hline $6^{\mathrm{o}}$ & 0.5918 & 0.7403 & -0.1485 & 0.5650 & 0.7135 \\
\hline
\end{tabular}




\subsection{NASA Transonic Cryogenic Wind Tunnel}

The same procedure of analysis, as described in previous section, has been applied here as well, also keeping the same airfoil, Mach number and analyzed angles of attack. Experimental conditions, comparing T-38 and NASA tunnels, on one side - slightly differ in the sense of Reynolds numbers which, for example for $\mathrm{M}=0.3$, are $\mathrm{Re}=4.4 \times 10^{6}$ in $\mathrm{T}-38$ and $\mathrm{Re}=$ $6.0795 \times 10^{6}$ in NASA tunnel. These Reynolds numbers are of the same order (4.4 and 6 million) and cannot introduce any substantial difference in the sense of flow patterns around the airfoil, comparing measurements in two different facilities. (It should also be noted that Reynolds number affects only experimental data, and not numerical results based on potential model, because it is inherently inviscid).

On the other hand, the difference in relative test section heights ( $h=6$ for T-38, and $h=4$ for NASA tunnel) suggests that for this reason, required corrections for NASA wind tunnel should generally be slightly larger, than for T-38.

Comparisons between experimental and numerically obtained pressure coefficient distributions for $\mathrm{M}=0.3$ and angles of attack of $\alpha=2^{\circ}, 4^{\circ}, 6^{\circ}$, are shown in Fig.8.

Figure 8 shows for angle of attack $\alpha=4^{\circ}$, agreements between experimental and calculated $\mathrm{Cp}$ are generally very good. For other two angles of attack, slight discrepancies exist in the aft domain and trailing edge, while the differences in $\Delta \mathrm{Cp}$ between upper and lower surface, considering experiment and calculations, are practically of the same order (and it should be remembered that $\Delta C p$ generates lift and moment). Keeping in mind that numerical models always "think" in the same way, while experimental data can be subject to inevitable small measurement errors, associated to the actual test run (explanation for certain oscillations on all measured $C p$ curves that can be hardly be explained otherwise), the comment considering obtained numerical values is the same as for T-38, and numerical results can be qualified as proper in the sense of the verification of the numerical model.

The calculated free flow values for analyzed angles of attack are compared with numerically obtained tunnel pressure distributions in Figure 9. These differences have been integrated and quantified in Table 2.

Figure 10 shows it is obvious that for NASA wind tunnel case, with smaller relative test section height than in T38 , required corrections for all angles of attack are proportionally larger, also with increasing tendency for higher angles.

Finally, the calculated values of $\Delta \mathrm{Cp}$ distributions have been superimposed to the measured pressure coefficient distributions, and the corrected pressure coefficients for NASA wind tunnel have been obtained, as shown in Figure 10.

Experimental lift coefficients after applied corrections (as global parameters) are shown in Tab.2. These values, obtained for $\mathrm{M}=0.3$ and angles of attack of $\alpha=2^{\circ}, 4^{\circ}, 6^{\circ}$, are practically the same as corrected values of lift coefficient for T-38 wind tunnel under the same nominal flow conditions, which is the expected outcome of the entire calculation and correction procedure, when established properly.

Final verification is obtained through the global comparison of lift coefficients from the two wind tunnels under the same nominal flight conditions, which after the applied corrections have practically the same values, although measured (uncorrected) lift coefficients were quite different, as shown in Table 3. Last column of this table represents values taken from reference [14] and which are considered as exact values. 


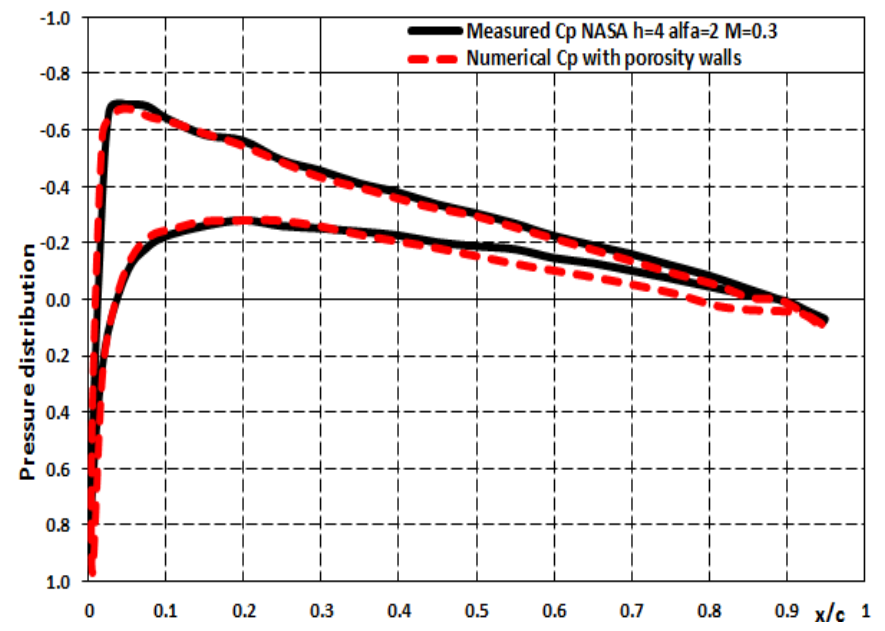

Figure 8: Measured and Calculation $C_{P}$ in NASA Wind Tunnel.

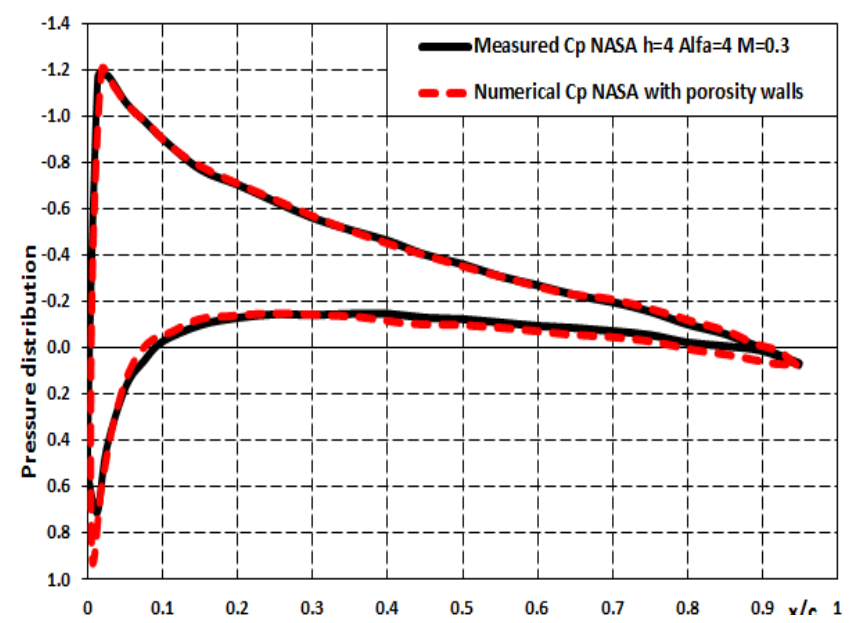

Figure 9: Measured and Calculation $C_{P}$ In NASA Wind Tunnel.

Table 2: Lift Coefficient Correction For NASA Wind Tunnel

\begin{tabular}{|c|c|c|c|c|c|}
\hline \multicolumn{5}{|c|}{ For NASA Wind Tunnel With Height $\mathbf{H}=\mathbf{4}, \mathbf{M}=\mathbf{0 . 3}$} \\
\hline Alfa & $\mathbf{C}_{\mathbf{L}}$ With Wall Effect & $\mathbf{C}_{\mathbf{L}}$ for Free Stream & $\boldsymbol{\Delta}_{\mathbf{L}}$ & $\mathbf{C}_{\mathbf{L}}$ Measured & $\mathbf{C}_{\mathbf{L}}$ After Correction \\
\hline $2^{\mathbf{0}}$ & 0.1740 & 0.2475 & -0.0735 & 0.1694 & 0.2429 \\
\hline $4^{\mathrm{o}}$ & 0.3715 & 0.4944 & -0.1229 & 0.3544 & 0.4773 \\
\hline $6^{\mathrm{o}}$ & 0.5686 & 0.7403 & -0.1717 & 0.5351 & 0.7068 \\
\hline
\end{tabular}




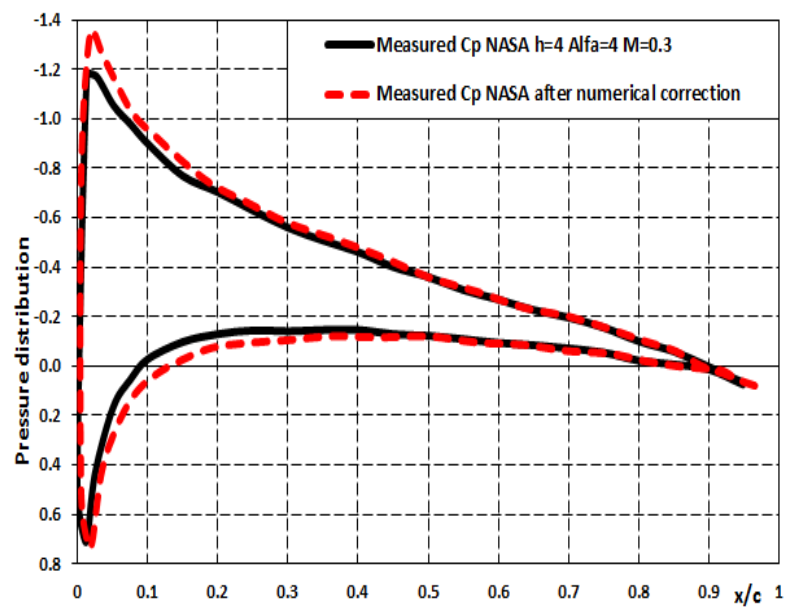

Figure 10: Measured $C_{p}$ After Correction in NASA

Wind Tunnel.

Table 3: Lift Coefficients from T-38 and NASA Wind Tunnels Before and After Applied Corrections for $\mathrm{M}=\mathbf{0 . 3}$

\begin{tabular}{|c|c|c|c|c|c|}
\hline Alfa & $\begin{array}{c}\text { T-38 } \mathbf{C}_{\mathbf{L}} \\
\text { Measured }\end{array}$ & $\begin{array}{c}\text { NASA C } \\
\text { Measured }\end{array}$ & $\begin{array}{c}\text { T-38 } \mathbf{C}_{\mathbf{L}} \text { After } \\
\text { Correction }\end{array}$ & $\begin{array}{c}\text { NASA } \mathbf{C}_{\mathbf{L}} \text { After } \\
\text { Correction }\end{array}$ & $\begin{array}{c}\text { Abbott } \mathbf{C}_{\mathbf{L}} \text { for } \\
\text { Free Stream }\end{array}$ \\
\hline $2^{\mathrm{o}}$ & 0.1900 & 0.1694 & 0.2444 & 0.2429 & 0.23 \\
\hline $4^{\mathrm{o}}$ & 0.3860 & 0.3544 & 0.4834 & 0.4773 & 0.45 \\
\hline $6^{\circ}$ & 0.5650 & 0.5351 & 0.7135 & 0.7068 & 0.65 \\
\hline
\end{tabular}

\section{CONCLUSIONS}

A panel method with linear vortex and constant source strength has been developed for the calculation interference of wall correction. The airfoil (NACA 0012) represented with enough number of the vortex withlinear strength segments placed inside test section. The linear vortex panels are mirrored due to the upper and lower test section walls. The porous walls simulated by sufficient number of constant source strength panels. For free stream (without walls) and inside test section (with walls effect) the pressure coefficient distribution around airfoil was calculated using linear vortex and the constant source strengths. The difference between pressure coefficient distribution withnumerical solution about airfoil with and without the presence of wind tunnel walls should be superimposed to the coefficient ofpressure distribution measured at the corresponding points. The resultant pressure coefficient distribution is employed to obtain the free stream lift coefficient for the same angle of attack and Mach number as in test section of wind tunnel. The corrections in this paper are directly applied to the coefficient ofpressure distribution.

The numerical method presented here has been verified by comparing the resultant lift coefficient correction with two classical methods well-known and wereobtained good agreements for two wind tunnel test sections.

The linear vortex and constant source strength panels enhance this numerical scheme and make it an efficient tool and reduces the computational time. This methodology is suitable for correcting measured data in $2 \mathrm{D}$ wind tunnel. In the future work, to three-dimension model this method will be extended.

\section{REFERENCES}

1. Abdullah, T. A.; Petrović, Z.; Stefanović, Z.; Kostić, I.; Isaković, J. Two-dimensional wind tunnel measurement corrections by the singularity method. // Tehnicki vjesnik-Technical Gazette. 22, 3(2015), pp. 557-565. https://doi.org/10.17559/TV20140214114718. 
2. Dawei, L.; Xin, X.; Qiang, L.; Xin, P.; Dehua C. Correction of model deformation effects for a supercritical wing in transonic wind tunnel. // Tehnicki vjesnik-Technical Gazette. 24, 6(2017), pp. 1647-1655. https://doi.org/10.17559/TV-20160525142932.

3. Ganzer, U. Adaptable wind tunnel walls for 2-D and 3-D model tests. // 12th Congress of the international council of the aeronautical sciences ICAS / Munich Germany, 1980. Proceedings, pp. 808816.

4. Meyer, O.; Blume, S.; Nitsche, W. Experimental investigations on the Adaptation accuracy of adaptive slots in Wind tunnel test section walls. // 23rd Congress of the international council of the aeronautical sciences ICAS / Toronto Canada, 2002. Proceedings, pp. 372.1372.10.

5. Sawada, H. Wind tunnel wall interference in a test section with ventilated walls. // 12th Congress of the international council of the aeronautical sciences ICAS / Munich Germany, 1980. Proceedings, pp. 823836.

6. Mokry, M.; Ohman, L. H. Application of the fast Fourier transform to two-dimensional wind tunnel wall interference. // Journal of Aircraft. 17, 6(1980), pp. 402-408. DOI: 10.2514/3.44666.

7. Kupper, A. Wall correction method with measured boundary conditions for low speed wind tunnel. // paper 13 in AGARD CP535, July 1994, pp. 13.113.10.

8. Mokry, M.; Digney, J. R.; Poole, R. J. D. Doublet-panel method for half-model wind-tunnel corrections. // Journal of Aircraft. 24, 5(1987), pp. 322-327. DOI: 10.2514/3.45448.

9. Beutner, T. J.; Celik, Z. Z.; Roberts, L. Determination of solid/Porous wall boundary conditions from wind tunnel data for computational fluid dynamics Codes. // paper 16 in AGARD CP-535, July 1994, pp. 16.116.19.

10. [Ashill, P. R.; Weeks, D. J. A method for determining wall-interference corrections in solid wall tunnels from measurements of static pressure at the walls. // AGARD-CP-335, May 1982, pp. 1.11.12.

11. Holt, D. R.; Hunt, B. The use of panel methods for the evaluation of subsonic wall interference. // Paper 2 in AGARD CP-335, May 1982, pp. 2.12.16.

12. Horstein, B. J. C.; Veldhuis, L. L. M. A New Hybrid Method to Correct for Wind Tunnel Wall-and Support Interference Online. // International Journal of Research and Scientific Innovation. 3, 10(2009), pp. 501508.

13. [13] Sheldahl, R. E.; Klimas, P. C. Aerodynamic Characteristics of Seven Symmetrical Airfoil Sections through 180-Degree Angle of Attack for Use in Aerodynamic Analysis of Vertical Axis Wind Turbines. // Sandia National Laboratories Energy Report: SAND80-2114; 1981.

14. Abbott, I. H.; von Doenhoff, A. E.; Stivers, Jr. L. S. Summary of Airfoil Data. // NACA Report No. 824. 1933.

15. Moore, A. W.; Wight, K. C. An Experimental Investigation of Wind-Tunnel Wall Conditions for Interference-Free Dynamic Measurements. Reports and Memoranda No. 3715" December, 1969.

16. Ladson, C. L.; Hill, A. S.; W. G. (n.d.). Pressure distributions from High Reynolds number transonic tests of an NACA 0012 airfoil in the Langley 0.3-meter transoniccryogenic tunnel. NASA Technical Memorandum 100526, 1987.

17. Goodman, T. R. The porous wall wind tunnel. Part II. Interference effect on a cylindrical body in a two-dimensional tunnel at subsonic speed. Cornell Aeronautical Lab. Report No. A.D-594-A-3. November, 1950.

18. Maeder, P. F. Investigation of the boundary condition at a perforated wall. Brown University Divn. of Engineering. Tech. Report WT-9. May, 1953.

19. Aleksandar Vitić. Experimental determination NACA 0012 characteristics in the T-38 wind tunnel two-dimensional test section. Scientific Technical Review, Vol. XLI, no.2, 1991. 
20. Veetil, Manoj, Spoorthi S. Prasad, and Abhay Vir Singh. "Test Section Blockage Corrections for Subsonic Open-Circuit Wind Tunnel." International Journal of Mechanical and Production Engineering Research and Development (IJMPERD) 9.2 (2019):645648.

21. Alrobaian, Abdulrahman A., S A Khan, and Mohammed Asadullah. "A New Approach to Low-Cost Open-Typed Subsonic Compressible Flow Wind Tunnel for Academic Purpose." International Journal of Mechanical and Production Engineering Research and Development (IJMPERD) 8.6 (2018):383394.

22. Pittala, Suresh, and T. Diriba. "Computational Fluid Dynamics Analysis of Impeller Design for A Pump." International Journal of Mechanical Engineering (IJME) 5.4 (2016):6374.

23. Govardhan, D., and B. Praveen. "Design And Analysis Of Two Throat Wind Tunnel." International Journal of Mechanical and Production Engineering Research and Development (IJMPERD) 7.4 (2017):381388. 